\title{
483.
}

\section{ON A PAIR OF DIFFERENTIAL EQUATIONS IN THE LUNAR THEORY.}

[From the Monthly Notices of the Royal Astronomical Society, vol. xxxII. (1871-72), pp. 201-206.]

I CONSIDER the differential equations

$$
\begin{aligned}
\frac{d}{d t} \frac{d \rho}{d t}-\rho\left(\frac{d v}{d t}\right)^{2}+\frac{1}{\rho^{2}} & =k m^{2} \rho\left\{\frac{1}{2}+\frac{3}{2} \cos (2 v-2 m t)\right\} \\
\frac{d}{d t}\left(\rho^{2} \frac{d v}{d t}\right) & =j m^{2} \rho^{2}\left\{-\frac{3}{2} \sin (2 v-2 m t)\right\}
\end{aligned}
$$

which when $j=k=1$ give the following equations in the lunar theory $(D=t-m t)$ :

$$
\begin{aligned}
\frac{1}{\rho}=1 & +\frac{1}{6} m^{2}-\frac{179}{288} m^{4}-\frac{97}{48} m^{5}-\frac{757}{162} m^{6}-\frac{4039}{432} m^{7}-\frac{34751189}{1990656} m^{8}-\frac{155067635}{4976640} m^{9} \\
& +\cos 2 D\left[m^{2}+\frac{19}{6} m^{3}+\frac{131}{18} m^{4}+\frac{383}{27} m^{5}+\frac{5105655}{20736} m^{6}+\frac{23140781}{622080} m^{7}\right. \\
& \left.+\frac{355021217}{9331200} m^{8}+\frac{27888590059}{34992000} m^{9}\right] \\
& +\cos 4 D\left[\frac{7}{8} m^{4}+\frac{2737}{480} m^{5}+\frac{162869}{7200} m^{6}+\frac{7554833}{108000} m^{7}+\frac{23889416723}{12960000} m^{8}+\frac{23352301255283}{5443200000} m^{9}\right] \\
& +\cos 6 D\left[\frac{219}{256} m^{6}+\frac{151339}{17920} m^{7}+\frac{29887443}{627200} m^{8}+\frac{98978623957}{444528000} m^{9}\right] \\
& +\cos 8 D\left[\frac{2701}{3072} m^{8}+\frac{70033633}{6021120} m^{9}\right],
\end{aligned}
$$

or as far as $m^{7}$,

$$
\begin{aligned}
\rho=1 & -\frac{1}{6} m^{2}+\frac{331}{288} m^{4}+\frac{83}{16} m^{5}+\frac{42775}{2592} m^{6}+\frac{4787}{108} m^{7} \\
& +\cos 2 D\left[-m^{2}-\frac{19}{6} m^{3}-\frac{125}{18} m^{4}-\frac{709}{54} m^{5}-\frac{485173}{20736} m^{6}-\frac{24487949}{622080} m^{7}\right] \\
& +\cos 4 D\left[-\frac{3}{8} m^{4}-\frac{1217}{480} m^{5}-\frac{74069}{7200} m^{6}-\frac{1749779}{54000} m^{7}\right] \\
& +\cos 6 D\left[-\frac{59}{256} m^{6}-\frac{126193}{53760} m^{7}\right],
\end{aligned}
$$

c. VII. 
$\left(\frac{1}{\rho}\right.$ is given by M. Delaunay only as far as $m^{5}$, the additional terms of $\frac{1}{\rho}$ and expression for $\rho$ were kindly communicated to me by Prof. Adams); and

$v=t$

$$
\begin{aligned}
& +\sin 2 D\left(\frac{11}{8} m^{2}+\frac{59}{12} m^{3}+\frac{893}{72} m^{4}+\frac{2855}{108} m^{5}+\frac{8304449}{165888} m^{6}\right. \\
& + \\
& \left.+\frac{102859909}{12 \frac{12}{460}} m^{7}+\frac{7596606727}{74649600} m^{8}-\frac{8051418161}{111974400} m^{9}\right) \\
& +\sin 4 D\left(\frac{201}{256} m^{4}+\frac{649}{120} m^{5}+\frac{6476233}{28800} m^{6}+\frac{31363361}{432000} m^{7}+\frac{123030377303}{414720000} m^{8}\right) \\
& +\sin 6 D\left(\frac{3715}{6144} m^{6}+\frac{664571}{107520} m^{7}\right)
\end{aligned}
$$

(Delaunay, t. II. pp. $815,836,845$ ).

To integrate the original equations write

$$
\begin{aligned}
& \rho=1+\rho_{1}+\rho_{2}+\ldots, \\
& v=t+v_{1}+v_{2}+\ldots,
\end{aligned}
$$

where the suffixes indicate the degrees in the coefficients $k, j$ conjointly: the equations for $\rho_{n}, v_{n}$ take the form

$$
\begin{aligned}
& \frac{d}{d t} \frac{d \rho_{n}}{d t}-3 \rho_{n}-2 \frac{d v_{n}}{d t}+V_{n}=Q_{n}, \\
& \frac{d}{d t}\left(\frac{d v_{n}}{d t}+2 \rho_{n}+U_{n}\right) \quad=P_{n},
\end{aligned}
$$

where $V_{n}, U_{n}, P_{n}, Q_{n}$ do not contain $\rho_{n}$ or $v_{n}$. From the second equation we have

$$
\frac{d v_{n}}{d t}+2 \rho_{n}+U_{n}=\Omega_{n}+\int P_{n} d t
$$

where $\Omega_{n}$ is a constant of integration, the integral $\int P_{n} d t$ containing only periodic terms; and then adding twice this to the first equation we have

$$
\frac{d}{d t} \frac{d \rho_{n}}{d t}+\rho_{n}+V_{n}+2 U_{n}=2 \Omega_{n}+Q_{n}+2 \int P_{n} d t
$$

which determines $\rho_{n}$; and substituting its value in the other equation we have $\frac{d v_{n}}{d t}$, and thence $v_{n}$; the constant $\Omega_{n}$ is determined so that $\frac{d v_{n}}{d t}$ may contain no constant term. We have

$$
\begin{aligned}
V_{1}= & 0 \\
V_{2}= & -\left(\frac{d v_{1}}{d t}\right)^{2}-2 \rho_{1} \frac{d v_{1}}{d t}+3 \rho_{1}^{2} \\
V_{3}= & -2 \frac{d v_{1}}{d t} \frac{d v_{2}}{d t}-2 \rho_{1} \frac{d v_{2}}{d t}-\rho_{1}\left(\frac{d v_{1}}{d t}\right)^{2} \\
& -2 \rho_{2} \frac{d v_{2}}{d t}+6 \rho_{1} \rho_{2}-4 \rho_{1}^{3}
\end{aligned}
$$

$\& c$.

$$
\begin{aligned}
& U_{1}=0 \\
& U_{2}=2 \rho_{1} \frac{d v_{1}}{d t}+\rho_{1}^{2} \\
& U_{3}=2 \rho_{1} \frac{d v_{2}}{d t}+\left(2 \rho_{2}+\rho_{1}^{2}\right) \frac{d v_{1}}{d t}+2 \rho_{1} \rho_{2}
\end{aligned}
$$

\&c. 


$$
\begin{aligned}
& Q_{1}=k m^{2}\left(\frac{1}{2}+\frac{3}{2} \cos 2 D\right), \\
& \begin{aligned}
Q_{2}=k m^{2} & \left\{3 v_{1} \sin 2 D+\rho_{1}\left(\frac{1}{2}+\frac{3}{2} \cos 2 D\right)\right\}, \\
Q_{3}=k m^{2} & \left\{-3 v_{2} \sin 2 D-3 v_{1}^{2} \cos 2 D\right. \\
& +\rho_{1} v_{1} \cdot 3 \sin 2 D \\
& \left.+\rho_{2}\left(\frac{1}{2}+\frac{3}{2} \cos 2 D\right)\right\}
\end{aligned}
\end{aligned}
$$

\&c.

$$
\begin{aligned}
P_{1}=j m^{2} & \left(-\frac{3}{2} \sin 2 D\right), \\
P_{2}=j m^{2} & \left(-3 v_{1} \cos D-3 \rho_{1} \sin 2 D\right), \\
P_{3}=j m^{2} & \left\{-3 v_{2} \cos 2 D+3 v_{1}^{2} \sin 2 D\right. \\
& -6 \rho_{1} v_{1} \cos 2 D \\
& \left.+\left(2 \rho_{2}+\rho_{1}^{2}\right) \cdot-\frac{3}{2} \sin 2 D\right\},
\end{aligned}
$$

\&c.

In particular attending to the values of $P_{1}, Q_{1}$ the equations for $\rho_{1}, v_{1}$ are in their original form

$$
\begin{aligned}
& \frac{d}{d t} \frac{d \rho_{1}}{d t}-3 \rho_{1}+2 \frac{d v_{1}}{d t}=k m^{2}\left(\frac{1}{2}+\frac{3}{2} \cos 2 D\right), \\
& \frac{d}{d t}\left(\frac{d v_{1}}{d t}+2 \rho_{1}\right)=j m^{2}\left(-\frac{3}{2} \sin 2 D\right),
\end{aligned}
$$

whence in the transformed form they are

$$
\frac{d v_{1}}{d t}+2 \rho_{1}=\Omega_{1}+\frac{3 j m^{2}}{4(1-m)} \cos 2 D,
$$

and

$$
\frac{d^{2} \rho_{1}}{d t^{2}}+\rho_{1}=2 \Omega_{1}+k m^{2}\left(\frac{1}{2}+\frac{3}{2} \cos 2 D\right)+\frac{\frac{3}{2} j m^{2}}{1-m} \cos 2 D
$$

Thus the constant term of $\rho_{1}$ is $2 \Omega_{1}+\frac{1}{2} k m^{2}$, giving in $\frac{d v_{1}}{d t}$ a constant term $-3 \Omega_{1}-k m^{2}$ this must vanish, or we have $\Omega_{1}=-\frac{1}{3} \mathrm{~km}^{2}$; and the equations thus become

$$
\begin{aligned}
& \frac{d v_{1}}{d t}+2 \rho_{1}=-\frac{1}{3} k m^{2}+\frac{3 j m^{2}}{4(1-m)} \cos 2 D, \\
& \frac{d^{2} \rho_{1}}{d t^{2}}+\rho_{1}=-\frac{1}{6} k m^{2}+\left(\frac{3}{2} k m^{2}+\frac{\frac{3}{2} j m^{2}}{1-m}\right) \cos 2 D,
\end{aligned}
$$

and then completing the integration

$$
\begin{aligned}
& \rho_{1}=-\frac{1}{6} k m^{2}+\left\{\frac{-\frac{3}{2} k m^{2}}{3-8 m+4 m^{2}}+\frac{-\frac{3}{2} j m^{2}}{(1-m)\left(3-8 m+4 m^{2}\right)}\right\} \cos 2 D, \\
& v_{1}=\quad\left\{\frac{\frac{3}{2} k m^{2}}{(1-m)\left(3-8 m+4 m^{2}\right)}+\frac{\frac{3}{8} j m^{2}\left(7-8 m+4 m^{2}\right)}{(1-m)^{2}\left(3-8 m+4 m^{2}\right)}\right\} \sin 2 D,
\end{aligned}
$$

which are the accurate values of $\rho_{1}$ and $v_{1}$.

Expanding as far as $m^{6}$ we have

$$
\begin{array}{ll}
\rho_{1}=k\left(-\frac{1}{6} m^{2}\right)+\cos 2 D\{ & k\left(-\frac{1}{2} m^{2}-\frac{4}{3} m^{3}-\frac{26}{9} m^{4}-\frac{160}{27} m^{5}-\frac{968}{81} m^{6}\right) \\
& \left.+j\left(-\frac{1}{2} m^{2}-\frac{11}{6} m^{3}-\frac{85}{18} m^{4}-\frac{575}{54} m^{5}-\frac{3661}{162} m^{6}\right)\right\}, \\
j=k \text { is } & =k\left(-m^{2}-\frac{19}{6} m^{3}-\frac{137}{18} m^{4}-\frac{895}{54} m^{5}-\frac{5597}{162} m^{6}\right),
\end{array}
$$

which for $j=k$ is 
and

$$
\begin{aligned}
& v_{1}=\quad \sin 2 D\left\{k\left(\frac{1}{2} m^{2}+\frac{11}{6} m^{3}+\frac{85}{18} m^{4}+\frac{575}{54} m^{5}+\frac{3661}{162} m^{6}\right)\right. \\
& \left.+j\left(\frac{7}{8} m^{2}+\frac{37}{12} m^{3}+\frac{589}{72} m^{4}+\frac{1037}{54} m^{5}+\frac{27331}{648} m^{6}\right)\right\}, \\
& \text { which for } j=k \text { is } \quad=k\left(\frac{11}{8} m^{2}+\frac{59}{12} m^{3}+\frac{1929}{72} m^{4}+\frac{8969}{27} m^{5}+\frac{41975}{648} m^{6}\right) \text {. }
\end{aligned}
$$

I have, not in general, but for the value $j=k$, calculated $\rho_{2}$ and $v_{2}$ as far as $m^{6}$ : I have not made the calculation for $\rho_{3}$ and $v_{3}$, but their values may be deduced from the foregoing values of $\rho, v$; the final expressions (when $j=k$ ) of $\rho,=1+\rho_{1}+\rho_{2}+\rho_{3}+\ldots$ and $v,=t+v_{1}+v_{2}+v_{3} \ldots$ are

$$
\begin{aligned}
& \rho=1 \\
& +k\left(-\frac{1}{6} m^{2}\right. \\
& +k^{2}\left(\quad \frac{331}{285} m^{4}+\frac{83}{16} m^{5}+\frac{5113}{288} m^{6}\right) \\
& +k^{3}\left(\quad-\frac{1621}{1296} m^{6}\right) \\
& +\cos 2 D\left\{k\left(-m^{2}-\frac{19}{6} m^{3}-\frac{137}{18} m^{4}-\frac{895}{54} m^{5}-\frac{55997}{162} m^{6}\right)\right. \\
& +k^{2}\left(\quad \frac{2}{3} m^{4}+\frac{31}{9} m^{5}+\frac{329}{27} m^{6}\right) \\
& \left.+k^{3}\left(\quad-\frac{2381}{2304} m^{6}\right)\right\} \\
& +\cos 4 D\left\{k^{2}\left(\quad-\frac{3}{8} m^{4}-\frac{1217}{480} m^{5}-\frac{76589}{7200} m^{6}\right)\right. \\
& +k^{3}( \\
& \left.\left.+\frac{7}{20} m^{6}\right)\right\} \\
& +\cos 6 D\left\{\dot{\kappa}^{3}\left(\quad-\frac{59}{256} m^{6}\right)\right\},
\end{aligned}
$$

and

$$
\begin{aligned}
& v=t \\
& +\sin 2 D\left\{k\left(\frac{11}{8} m^{2}+\frac{59}{12} m^{3}+\frac{929}{72} m^{4}+\frac{896}{27} m^{5}+\frac{41975}{648} m^{6}\right)\right. \\
& +k^{2}\left(\quad-\frac{1}{2} m^{4}=\frac{41}{12} m^{5}-\frac{43}{3} m^{6}\right) \\
& \left.+k^{3}\left(\quad-\frac{783}{2048} m^{6}\right)\right\} \\
& +\sin 4 D\left\{k^{2}\left(\quad \frac{201}{256} m^{4}+\frac{649}{120} m^{5}+\frac{665263}{28800} m^{6}\right)\right. \\
& \left.+k^{3}\left(-\frac{49}{80} m^{6}\right)\right\} \\
& +\sin 6 D\left\{k^{3}\left(\quad+\frac{3715}{6144} m^{6}\right)\right\} \text {; }
\end{aligned}
$$

which for $k=1$ agree with the foregoing formulæ (verifying them as far as $m^{5}$ ); the present formulæ exhibit the manner in which the expressions depend on the several powers of the disturbing force. 